\title{
CAMBIOS A FINA ESCALA DE LA ARIDEZ EN LA COMUNIDAD VALENCIANA ENTRE 1948 Y 2011
}

\author{
Juan Javier MIRÓ ${ }^{1}$, María José ESTRELA², Vicente CASELLES ${ }^{1}$ \\ ${ }^{1}$ Departament de Física de la Terra i Termodinàmica, Facultat de Física, Universitat de València, \\ Doctor Moliner 50, Burjassot, Valencia 46100, Spain. \\ ${ }^{2}$ Departament de Geografia, Facultat de Geografia i Història, Universitat de València, Avda. \\ Blasco Ibáñez 28, Valencia 46010, Spain. \\ Javier.Miro-Perez@uv.es, Maria.Jose.Estrela@uv.es, vicente.caselles@uv.es
}

\section{RESUMEN}

Recientes avances en downscaling estadístico e interpolación espacial de temperaturas han resultado en la disponibilidad del dataset SDSITVC (Statistical Downscaling and Spatial Interpolation of Temperatures for the Valencian Community), con una resolución espacial de $90 \mathrm{~m}$ y periodo completo de 1948-2011, para la Comunidad Valenciana y áreas limítrofes. El presente trabajo busca relacionar dicha interpolación de temperaturas con otra de precipitaciones que, al igual que el SDSITVC, sea apta para el análisis de tendencias entre 1948 y 2011. Hasta la fecha no hay aún disponible una interpolación de precipitaciones con la misma resolución espacial, si bien se ha podido utilizar el dataset Spain02_v3, cuya resolución original es de $20 \mathrm{~km}$, como alternativa razonable para poder realizar una primera estimación espacial de cambios de la aridez en curso. Basándose en estudios previos sobre el SDSITVC que establecen la idoneidad de un periodo de referencia (1948-1979) y un periodo de testeo del cambio (1997-2011), el estudio determina cambios en la aridez entre los dos periodos según la aplicación de tres índices bioclimáticos simples y conocidos: Dantín - Revenga, Emberger, y Vernet. Se presenta una cartografía del cambio acontecido en dichos índices para la Comunidad Valenciana. Los resultados indican que ya habría habido cambios apreciables hacia aumentos de la aridez entre el periodo de referencia y el de testeo, particularmente hacia el interior y zonas altas.

Palabras clave: Cambio climático, aridez, SDSITVC, Spain02_v3, Dantín Revenga, Emberger, Vernet.

\begin{abstract}
Recent advances in statistical downscaling and spatial interpolation of temperatures have resulted in the availability of the SDSITVC dataset (Statistical Downscaling and Spatial Interpolation of Temperatures for the Valencian Community). This complies a spatial resolution of $90 \mathrm{~m}$ and full 1948-2011 period, for the Valencian Community and surrounding areas. This paper seeks to relate this temperature interpolation with other for precipitation in the same way suitable for the analysis of trends between 1948 and 2011. To date there is no still available a spatial interpolation of precipitation with the same resolution of SDSITVC. While it has been possible to use the Spain02_v3 dataset, whose native resolution is $20 \mathrm{~km}$, as a reasonable alternative to a first spatial estimation of aridity changes in process. According to previous studies on
\end{abstract}


the SDSITVC, a reference period (1948-1979) and a testing period (1997-2011) are established. The study determined changes in aridity between the two periods depending on the application of three simple and well-known bioclimatic indices: Dantin - Revenga, Emberger, and Vernet. A cartography of the change happened in these indexes for Valencian Community is presented. The results indicate that there would have already been substantial changes towards aridity increases between the reference and test periods, particularly towards the interior and upland areas.

Key words: Climate change, aridity, SDSITVC, Spain02_v3, Dantín - Revenga, Emberger, Vernet.

\section{INTRODUCCIÓN}

Son múltiples los estudios que ya han constatado tendencias generales al cambio climático en los últimos decenios sobre la Península Ibérica. Se han determinado así tendencias al aumento de las temperaturas en el conjunto de la Península (p. ej., Brunet et al., 2007; Del- Río et al, 2012); y también cambios incipientes en los patrones de precipitación (Millán et al, 2005; Muñoz-Díaz y Rodrigo, 2006; Estrela et al., 2010; Miró et al., 2010; González-Hidalgo et al., 2010; Gallego et al., 2011). Sin embargo, se ha señalado que las mayores incertidumbres sobre los impactos del cambio climático se encuentran en los niveles regional y local (IPCC, 2013), lo cual es especialmente cierto para las regiones mediterráneas. De modo que se hacen necesarios estudios sobre el cambio climático y sus impactos en dichas escalas.

Para el caso concreto de la Comunidad Valenciana tenemos al respecto avances recientes. Se trata de la disponibilidad del dataset SDSITVC (Statistical Downscaling and Spatial Interpolation of Temperatures for the Valencian Community), que ha permitido determinar patrones de cambio en las temperaturas a fina escala $(90 \mathrm{~m})$ entre 1948 y 2011 (Miró, 2014; Miró et al., 2015 y 2016a). Los patrones de cambio revelados señalan un proceso ya en curso de acusado ascenso térmico en las zonas altas y montañosas del interior de la región, que no se habría constatado de tener sólo en cuenta las series observadas más largas y sin lagunas, y con una posición más bien litoral (Miró et al., 2015 y 2016a). Se trata además de cambios no proyectados, sino ya acontecidos en las últimas décadas.

Por otro lado, estos cambios en curso nos llevan a la cuestión de hasta qué punto están ya hoy en día forzando un cambio en las condiciones bioclimáticas y, por tanto, comprometiendo el equilibrio de biotopos y recursos dependientes del clima. Precisamente, la falta de herramientas a fina escala para evaluar tendencias al cambio ha resultado hasta ahora en la falta de cartografías del cambio aplicadas a los propios índices bioclimáticos al uso. La disponibilidad del SDSITVC sí ha permitido, en este caso, evaluar el cambio bioclimático potencial dependiente de las temperaturas, a través del índice de Termicidad de Rivas-Martínez (Rivas-Martínez, 2008; Miró et al., 2016b). Dicha evaluación ha determinado que actualmente se está produciendo una acusada regresión potencial de termotipos bioclimáticos que están en posición finícola en las montañas de la región (p. ej. Supramediterráneo, Supratemplado, Orotemplado), de modo que algunos horizontes termotípicos están incluso en trance de desaparecer (Miró et al., 2016b). 
Sin embargo, los referidos estudios relativos al SDSITVC sólo han podido evaluar elementos de cambio dependientes de la temperatura, siendo la precipitación el otro elemento básico que determina el cálculo de la mayoría de índices bioclimáticos. Por supuesto, es la evapotranspiración la que determinará un cálculo más ajustado, por cuanto la aridez es básicamente una función de la evapotranspiración y la precipitación. Pero, en su ausencia, son múltiples los índices que desde distintas maneras abogan por el uso de la precipitación y la temperatura como principales determinantes para una clasificación aceptable de la aridez, basándose en el supuesto de que con la temperatura aumenta correlativamente la evapotranspiración (Capel Molina, 2000).

Hasta la fecha todavía no está disponible una interpolación espacial de precipitaciones con las mismas características y resolución espacial-temporal que el SDSITVC, por lo que, a priori, no puede realizarse una caracterización de tendencias para las precipitaciones en una escala con el mismo nivel de detalle. Ello a pesar de tener una densa red de observatorios pluviométricos, pero cuyas tendencias no son comparables entre sí al tratarse de series de duración temporal, presencia de datos ausentes, homogeneidad, etc., muy heterogéneos.

Una alternativa es el uso de datasets interpolados de menor resolución espacial pero con una cobertura temporal similar, y validez para su uso en estudios de tendencias. Al respecto está disponible el producto 'Spain02' elaborado conjuntamente por AEMET y el Grupo de Meteorología de Santander para toda la Península Ibérica y Baleares. Éste existe en 3 versiones. La v2 y v3 (Herrera et al., 2012a, 2012b) tienen una resolución espacial de $0.2^{\circ}(\approx 20 \mathrm{~km})$ y cobertura temporal de $1950 \mathrm{a}$ 2008 (extendida a 2011). Por su parte, la v4 (resolución de $0.11^{\circ}$ ) no nos interesa aquí ya que se inicia en 1973. Aunque la versión v2 comprende un mayor número de observatorios (más de 2700 en la Península), la v3 se realizó y testó específicamente para poder ser usada en análisis de tendencias, al basarse sólo en las series más largas (más de 800 observatorios).

El presente estudio tiene como objetivo presentar cartográficamente cambios potenciales en la aridez que ya habrían acontecido en los últimos decenios en la Comunidad Valenciana, valiéndose para ello de los datasets SDSITVC (temperaturas) y Spain02_v3 (precipitaciones). Los cambios en la aridez se determinarán a través del cálculo de tres índices conocidos para un periodo de referencia y un periodo de testeo. Éstos son: Dantín-Revenga, Emberger y Vernet.

\section{DATOS Y MÉTODOS}

\subsection{Datasets empleados}

El dataset SDSITVC (Miró, 2014; Miró et al., 2015, 2016a y 2016b) es el empleado como fuente de temperaturas, y el dataset Spain02_v3.1 (Herrera et al., 2012a y 2012b) el empleado como fuente de precipitaciones. El primero abarca de 1948 a 2011 y el segundo de 1950 a 2008 (extendido a 2011). Se elige la versión v3 (Spain02_v3.1) dado que es la más adecuada para su uso en análisis de tendencias. Ello es porque se basa en una red de más de 800 estaciones peninsulares con al menos 40 años y el $90 \%$ de los datos completos en el periodo que abarca (Herrera et al., 2012a y 2012b). Por su parte, el SDSITVC se basa en un downscaling estadístico sobre más de 300 series observadas en la región concreta de estudio, que proporciona características 
homogéneas a todos los datos desde 1948 a 2011, y patrones de cambio térmico bien definidos y espacialmente homogéneos con respecto a las características geográficas de cada punto (Miró, 2014; Miró et al., 2015 y 2016a). Ello con errores inferiores a $1^{\circ} \mathrm{C}$ en validación cruzada.

Dado que la resolución nativa del dataset SDSITVC es de 90 m en coordenadas UTM (huso 30), se ha tenido que ajustar a dicha resolución el dataset Spain02_v3.1, originariamente a una resolución mucho más baja $(\approx 20 \mathrm{~km})$. Para ello se ha re-interpolado la información del dataset Spain02_v3.1 a la misma resolución del SDSITVC utilizando el procedimiento de Mínima Curvatura, en aras de preservar lo más posible las relaciones espaciales originales del dataset con menor resolución.

\section{2. Índices aplicados}

Aunque existe un número amplio de índices propuestos para evaluar la aridez, se han aplicado tres, siguiendo un criterio de mayor a menor sencillez, sin perder de vista una buena relación entre efectividad y facilidad de implementación de todos ellos (sólo requieren temperaturas y precipitaciones). También, estos tres han sido, o bien ideados para el ámbito ibérico, o bien aplicados con buenos resultados en dicho ámbito.

El primero es el índice de Martín Cereceda y Revenga (Dantín Cereceda y Revenga Carbonell, 1942). Similar al índice de Martonne ó Lang, relaciona simplemente valores anuales medios de pluviometría y temperaturas, siendo ideado para su aplicación al ámbito ibérico. La fórmula aplicada para el índice de Martín y Revenga $(i D R)$ es:

$$
i D R=\frac{100 T}{P}
$$

donde $T$ es la temperatura media anual $\left({ }^{\circ} \mathrm{C}\right)$ y $P$ la precipitación total media anual (mm). La tabla 1 muestra la clasificación que se deriva el $i D R$.

\begin{tabular}{|c|c|}
\hline$i D R$ & TIPOS \\
\hline 0 a 2 & Húmedo \\
\hline 2 a 3 & Semiárido \\
\hline 3 a 6 & Árido \\
\hline$>6$ & Subdesértico \\
\hline
\end{tabular}

Tabla 1: Clasificación del grado de aridez según el Índice de Dantín-Revenga.

El segundo es el índice de Emberger (Emberger, 1930), que está ideado para regiones mediterráneas, y se ha considerado un buen indicador biogeográfico en el Sureste peninsular (Condés y García Robredo, 2012). En este caso, además de la precipitación total, introduce la evaluación de un contraste térmico entre las temperaturas anuales más frías y las más cálidas. La fórmula para el índice de Emberger $(i E)$ que aquí se aplica es la versión que aparece en Rivas-Martínez (2008): 


$$
i E=\frac{100 P}{M i^{2}-m i^{2}}
$$

donde $P$ es la precipitación total media anual $(\mathrm{mm}), M i$ la temperatura media $\left({ }^{\circ} \mathrm{C}\right)$ de las máximas del mes más cálido y $m i$ la temperatura media de las mínimas del mes más frío. Al expresar el valor $M i$-mi una amplitud térmica extrema anual, se idea como un factor proporcional al rango de evaporación. La tabla 2 muestra la clasificación del $i E$.

\begin{tabular}{|c|c|}
\hline$i E$ & TIPOS \\
\hline$>90$ & Húmedos \\
\hline 50 a 90 & Mediterráneo Subhúmedo \\
\hline 30 a 50 & Mediterráneo Semiárido \\
\hline 0 a 30 & Mediterráneo Árido \\
\hline
\end{tabular}

Tabla 2: Clasificación del grado de aridez según el Índice de Emberger.

El tercer índice aplicado es el de Vernet (Vernet y Vernet, 1966). Este índice va más allá de los anteriores al incorporar la importancia que el estrés hídrico estival tiene en el cálculo de la aridez, y que puede verse enmascarado en las medias o totales anuales. Pondera así las temperaturas máximas estivales, la precipitación estival y la diferencia de precipitación entre la estación más seca y la más húmeda del año. Aunque es menos conocido que los anteriores, se ha demostrado su validez en sectores montanos ibéricos, por ejemplo en Sánchez Palomares et al. (2001). Se define del siguiente modo:

$$
i V=100\left(\frac{H-h}{P}\right)\left(\frac{M v}{P v}\right)
$$

donde $P$ es la precipitación total media anual $(\mathrm{mm}), P v$ la precipitación total media del verano, $H$ la precipitación total media del trimestre más lluvioso del año, $h$ la precipitación total media del trimestre más seco del año, y $M v$ la temperatura máxima media $\left({ }^{\circ} \mathrm{C}\right)$ del verano. La tabla 3 muestra la clasificación derivada del $i V$.

\begin{tabular}{|c|c|}
\hline$i V$ & TIPOS \\
\hline$>2$ & Continental \\
\hline 0 a 2 & Oceánico-Continental \\
\hline-1 a 0 & Oceánico \\
\hline-2 a -1 & Pseudo-oceánico \\
\hline-3 a -2 & Oceánico-Mediterráneo \\
\hline-4 a -3 & Submediterráneo \\
\hline$<-4$ & Mediterráneo \\
\hline
\end{tabular}

Tabla 3: Clasificación del grado de aridez según el Índice de Vernet. 


\subsection{Periodos de contraste}

Se ha realizado el cálculo de los índices de aridez para un periodo de referencia y otro de testeo o contraste. Estos periodos se han delimitado sobre la base de los resultados obtenidos para las tendencias de temperatura reportados en Miró et al. (2015 y 2016a), y cambios en los horizontes termotípicos (Miró et al., 2016b). Se establece así como periodo de referencia el comprendido entre 1948 y 1979 (periodo aún sin cambios destacados en los patrones de temperatura). Tras un acusado incremento térmico entre 1980 y 1998, en los últimos 15 años de la serie se asienta en la región un cambio de patrones térmicos en torno a un nuevo estado térmico general más cálido. Se toma así como periodo de testeo los últimos 15 años disponibles (1997-2011). Dado que el dataset Spain02_v3.1 se inicia en 1950, para el caso de las precipitaciones se ha utilizado el periodo de referencia 1950-1979.

\section{RESULTADOS}

El resultado para los tres índices analizados, con su distribución espacial en el periodo de referencia y el periodo de testeo, se muestra en las figuras $1(i D R), 2(i E)$ y $3(i V)$.

Respecto del Índice de Dantín-Revenga (Fig.1) se aprecian amplias zonas donde potencialmente ha aumentado la aridez. Destaca sobre todo que grandes partes del interior Sur de Valencia han cambiado su condición 'semiárida' a 'árida'. También en parte del Valle del Turia. Por su parte se han reducido bastante los reductos catalogados como 'húmedos y subhúmedos' en la parte analizada del Sistema Ibérico (Gúdar, Javalambre, estribaciones orientales de los Montes Universales). Sólo aparecen dos áreas donde no se aprecian aumentos en la aridez, como es el extremo Norte de Castellón, y el polo subhúmedo entre el Nordeste de Alicante y Sureste de Valencia, relacionado este último con los irregulares temporales de levante. En este último caso incluso se aprecia cierto incremento del polo subhúmedo y su gradiente con el Sur de Alicante. En general los cambios son mayores hacia el interior con respecto al litoral.

Respecto del Índice de Emberger (Fig. 2) se aprecian resultados similares. Incluso se acusa más el cambio en la mayor parte del interior de Valencia y el Alto Vinalopó (Alicante). De este modo en el periodo de testeo comienza a asomar el tipo 'Mediterráneo Árido' en los límites del interior Sur de Valencia (Cabriel, Sur de Ayora) y partes del Valle del Vinalopó (Alicante), e incluso el litoral central de Alicante. El tipo 'Mediterráneo semiárido' también habría avanzado, a costa del 'Mediterráneo subhúmedo', en el Valle del Turia y resto del centro e interior de Valencia.

Las zonas con bioclimas potencialmente húmedos del Sistema Ibérico se habrían reducido en más de un $80 \%$. Pero, de nuevo, las excepciones a estos aumentos en la aridez son el tercio Norte de Castellón y el sector del extremo Nordeste de Alicante y Sureste de Valencia.

Respecto del Índice de Vernet (Fig. 3), a diferencia de los anteriores, no muestra el polo subhúmedo del Nordeste de Alicante - Sureste de Valencia, debido a que se trata de un sector con un fuerte mínimo pluviométrico estival coincidiendo con altas temperaturas, característica que proporciona una fuerte mediterraneidad según el sistema de Vernet. Es por ello que también la mayor parte de la región cae dentro del tipo mediterráneo. 


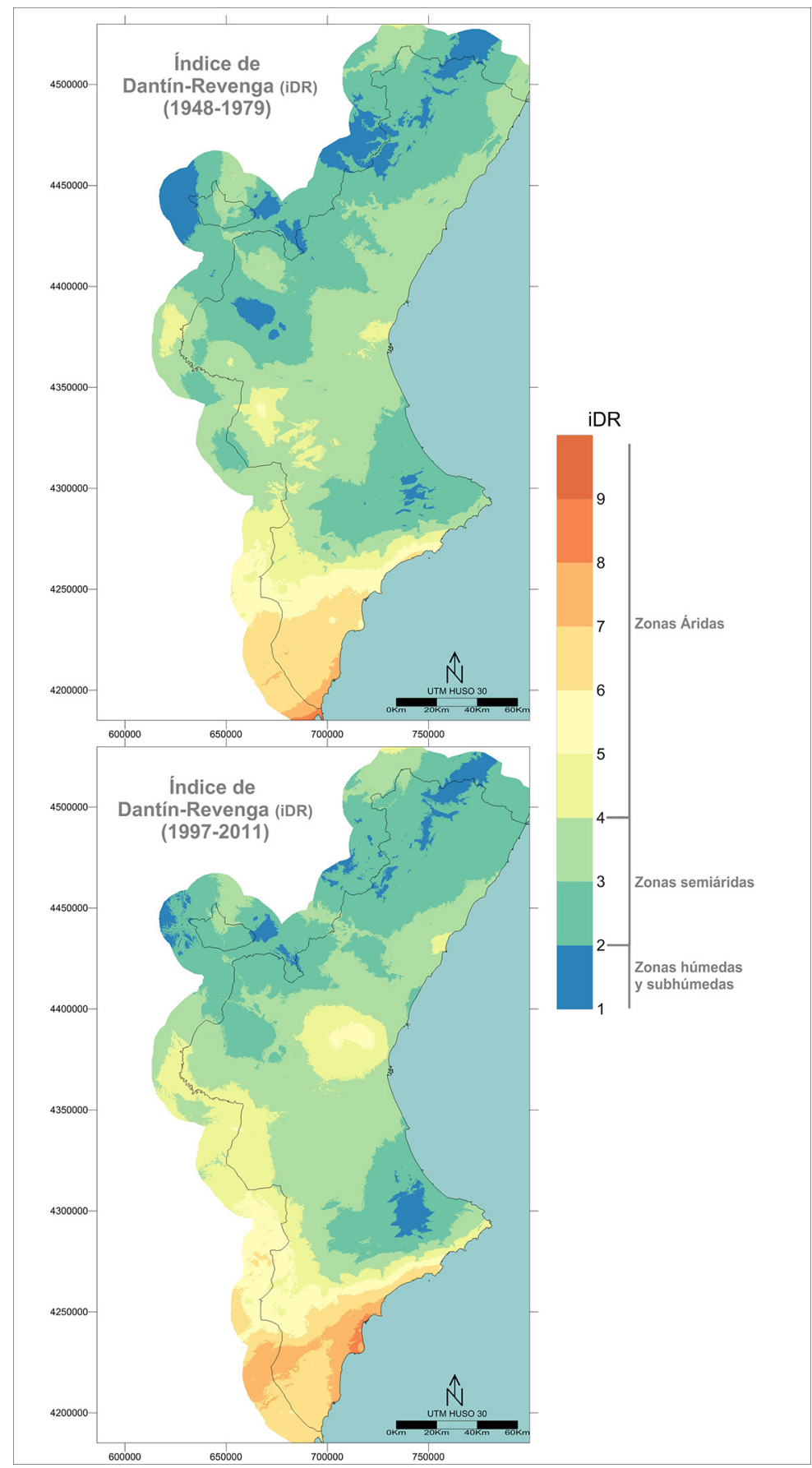

Figura 1: Grado de aridez según el Índice de Dantín-Revenga para los periodos de referencia $y$ de testeo 


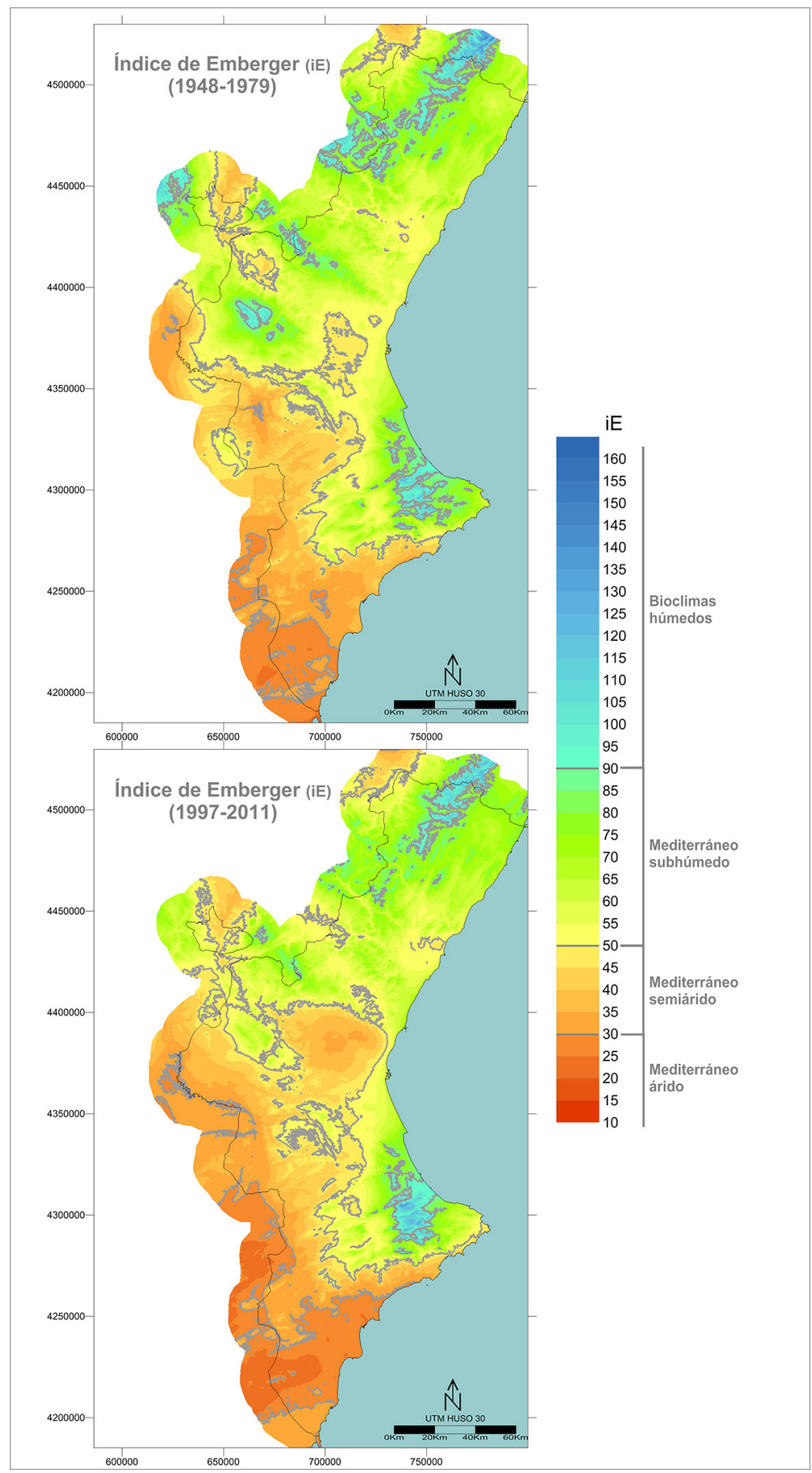

Figura 2: Grado de aridez según el Índice de Emberger para los periodos de referencia y de testeo 


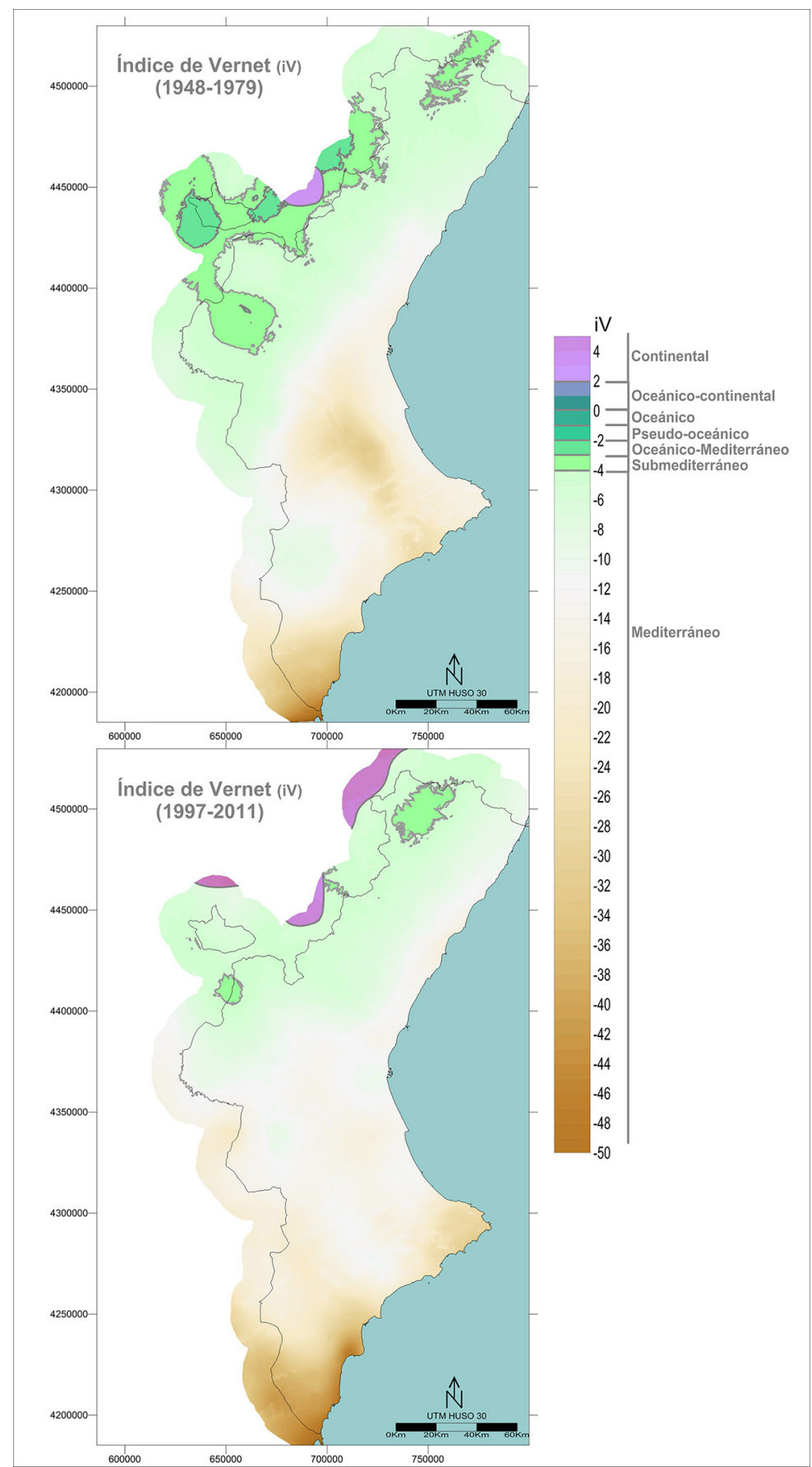

Figura 3: Grado de aridez según el Índice de Vernet para los periodos de referencia y de testeo 
Sin embargo, en el periodo de referencia aparecen bien definidos sectores bajo el tipo ‘Submediterráneo’ en la parte del Sistema Ibérico (interior de la mitad Norte), que derivan en 'Oceánico-Mediterráneo' en los sectores más elevados. Sin embargo, estos últimos han desaparecido potencialmente en el periodo de testeo, y el 'Submediterráneo’ casi también, a excepción del interior más al Norte de Castellón.

Es de notar también cambios en el grado de continentalidad hacia el interior del Sistema Ibérico, apareciendo en el periodo de referencia el tipo 'Continental' hacia la cabecera del Mijares, ya en Teruel. Este tipo 'Continental' incrementa su superficie en el periodo de testeo a nuevas zonas interiores del Sistema Ibérico.

\section{CONCLUSIONES}

El cálculo del grado de aridez en la Comunidad Valenciana a través de tres índices distintos, y utilizando los datasets SDSITVC (temperaturas) y Spain02_v3.1 (precipitaciones), ha determinado que ya se han producido cambios potenciales entre un periodo de referencia (1948-1979) y otro de testeo (1997-2011). La aridez habría aumentado en general más en el interior que en el litoral. Destaca la degradación de las condiciones semiáridas en el interior Sur de la Comunidad. Pero también la reducción de condiciones húmedas y subhúmedas en el Sistema Ibérico, en favor de una mayor mediterraneidad o bien de condiciones secas continentales (áreas más interiores). Se desmarcan de estas tendencias el tercio Norte de Castellón, el polo subhúmedo al Noreste de Alicante - Sureste de Valencia, y gran parte del mismo litoral central y Norte, áreas cuya precipitación está ligada a temporales de levante muy irregulares.

Estas tendencias sugieren una fuerte conexión con resultados obtenidos en la región respecto a diferentes tendencias según el tipo sinóptico ligado a la precipitación. En concreto, una tendencia al descenso de precipitaciones asociadas a frentes atlánticos de relativo mayor peso en el interior, que en el litoral se compensa por un aumento del peso relativo de las precipitaciones de origen mediterráneo (Millán, et al., 2005; Estrela et al., 2010; Miró et al., 2010; Miró et al., 2015). A ello se suma un cambio térmico mayor en las zonas altas del interior que en el litoral (Miró et al., 2015, 2016a y 2016b).

No obstante, cabe tener en cuenta que el dataset de precipitación utilizado puede no llegar a identificar los patrones de cambio más locales, como sí lo hace el SDSITVC para las temperaturas. Por ello es necesaria la creación de un dataset de mayor resolución para la precipitación que sí pueda contemplar esos cambios más locales.

\section{AGRADECIMIENTOS}

Este trabajo se ha realizado gracias al programa PROMETEOII (2014/086) de la Comunidad Valenciana, y el proyecto CGL2015- 64268-R (MINECO/FEDER, UE) del Ministerio de Economía y Competitividad y el FEDER.

\section{REFERENCIAS}

Brunet, M., et al. (2007). Temporal and spatial temperature variability and change over Spain during 1850-2005. J. Geophys. Res. D: Atmos., 112, D12117, doi: 10.1029/2006JD008249 
Capel Molina, J.J. (2000). El clima de la Península Ibérica. Editorial Ariel, colección Ariel Geografía. Barcelona, 281 p.

Condés, S. y García-Robredo, F. (2012). An empirical mixed model to quantify climate influence on the growth of Pinus halepensis Mill. stands in South-Eastern Spain. For. Ecol. Manage. 284: 59-68, doi:10.1016/j.foreco.2012.07.030

Dantín Cereceda, J. y Revenga Carbonell, A. (1941). Las líneas y las zonas ixóseras de España, según los índices termopluviométricos. Avance al estudio de la aridez en España. Estudios Geográficos, 2, 35-91.

Del-Río, S., Cano-Ortiz, A., Herrero, L. y Penas, A. (2012). Recent trends in mean maximum and minimum air temperatures over Spain (1921-2006). Theor. Appl. Climatol., 109, 605-626, doi: 10.1007/s00704-012-0593-2.

Emberger, L. (1930). La végétation de la région méditerranéenne: essai d'une classification des groupements végétaux. Revue Générale de Botanique, 42 (641662), 705-721

Estrela, M.J., Miró, J.J., Pastor, F. y Millán, M. (2010). Frontal Atlantic rainfall component in the Western Mediterranean Basin. Variability and spatial distribution. ESF-MedCLIVAR Workshop on "Hydrological, socioeconomic and ecological impacts of the North Atlantic Oscillation in the Mediterranean region". Zaragoza (Spain), 24-27 May 2010.

Gallego, M.C. et al (2011). Trends in frequency indices of daily precipitation over the Iberian Peninsula during the last century. J. Geophys. Res. D: Atmos., 116, D02109, doi:10.1029/2010JD014255

Gonzalez-Hidalgo, J.C., Brunetti, M. y de Luis, M. (2010). Precipitation trends in Spanish hydrological divisions, 1946-2005. Climate Res., 43, 215-228, doi: $10.3354 / \mathrm{cr} 00937$

Herrera et al. (2012a). Development and Analysis of a 50 year high-resolution daily gridded precipitation dataset over Spain (Spain02). Int. J. Clim., 32, 74-85, doi: 10.1002/joc. 2256

Herrera et al. (2012b). New versions of Spain02: Daily precipitation and temperature data for downscaling studies. $8^{\circ}$ Congreso Internacional de la AEC.

IPCC, (2013). Climate Change 2013: The Physical Science Basis. Contribution of Working Group I to the Fifth Assessment Report of the Intergovernmental Panel on Climate Change [Stocker, T.F., D. Qin, G.-K. Plattner, M. Tignor, S.K. Allen, J. Boschung, A. Nauels, Y. Xia, V. Bex and P.M. Midgley (eds.)]. Cambridge University Press, Cambridge. United Kingdom and New York, NY, USA, 1535 pp, doi: 10.1017/CBO9781107415324

Millán, M., Estrela, M.J. y Miró, J.J. (2005). Rainfall components: variability and spatial distribution in a mediterranean area (Valencia Region). J. Clim, 18 (14), 2682-2705, doi:10.1175/JCLI3426.1

Miró, J.J., Estrela, M.J., Pastor, F. y Millán, M. (2010). Análisis comparativo de tendencias en la precipitación, por distintos inputs, entre los dominios hidrológicos del Segura y del Júcar (1958-2008). Investigaciones Geográficas, 49, 129-157, doi: 10.14198/INGEO2009.49.07 
Miró J.J. (2014). Downscaling estadístico de series climáticas mediante redes neuronales: Reconstrucción en alta resolución de la temperatura diaria para la Comunidad Valenciana. Interpolación espacial y análisis de tendencias (19482011). Tesis Doctoral. Universidad de Alicante, Fundación Centro de Estudios Ambientales del Mediterráneo, y Universidad de Valencia. 523p., doi: 10.13140/ RG.2.1.2059.1523

Miró, J.J., Estrela, M.J. y Olcina-Cantos, J. (2015). Statistical downscaling and attribution of air temperature change patterns in the Valencia region (1948-2011). Atmos. Res., 156: 189-212, doi:10.1016/j.atmosres.2015.01.003

Miró, J.J., Estrela, M.J. y Olcina-Cantos, J. (2016a). Reconstrucción de la señal térmica local en la Comunidad Valenciana entre 1948 y 2011 a partir de un downscaling estadístico mediante una red neuronal artificial: Detección de patrones locales de cambio. Boletín de la Asociación de Geógrafos Españoles, 70, 113-147 (eng:471475), doi:10.21138/bage.2165

Miró, J.J., Estrela, M.J., Caselles, V. y Olcina-Cantos, J. (2016b). Fine-scale estimations of bioclimatic change in the Valencia region, Spain. Atmos. Res., In Press, Accepted Manuscript, doi:10.1016/j.atmosres.2016.05.020

Muñoz-Díaz, D. y Rodrigo, F.S. (2006). Seasonal rainfall variations in Spain (19122000) and their links to atmospheric circulation. Atmos. Res., 81 (1), 94-110, doi:10.1016/j.atmosres.2005.11.005

Rivas-Martínez, S. (2008). Global Bioclimatics, Internet: www.globalbioclimatics. org

Sánchez Palomares, O., Carretero, M.P. y Sarmiento, L.A. (2001). Definición y cartografía de las áreas potenciales fisiográfico-climáticas de los alcornocales catalanes (Quercus suber L.). In, Actas del III Congreso Forestal Español, vol. 1 y 2, Granada: 271-277.

Vernet, J.L. y Vernet, P.H. (1966). Sur un indice bioclimatique applicable aux climats de la France. Naturalia Monspeliensia, Série Botanique 17, 253-262 